\title{
On physical interpretation of 2D time-correlations regarding time delay velocities and eddy
}

shaping.

N. Fedorczak, ${ }^{1,2}$ P. Manz, ${ }^{1,3}$ S.C. Thakur, ${ }^{1}$ M. Xu, ${ }^{1}$ G.R. Tynan, ${ }^{1}$ G.S. Xu, ${ }^{4}$ and S.C. Liu ${ }^{4}$

${ }^{1)}$ Center for Momentum Transport and Flow Organization, University of California at San Diego, San Diego, California 92093, USA

${ }^{2)}$ Commissariat à l'Energie Atomique et aux Energies Alternatives, Institut de Recherche pour la Fusion Magnétique Controlée, F-13108 Saint-Paul-Lez-Durance, France ${ }^{\text {a) }}$

3) Max-Planck-Institut für Plasmaphysik, Association Euratom-IPP, 85748Garching, Germany

4) Institute of Plasma Physics, Chinese Academy of Sciences, Hefei 230031, People's Republic of China

Time delay estimation (TDE) techniques are frequently used to estimate the flow velocity from fluctuating measurements. Tilted structures carried by the flow lead to misinterpretation of the time delays in terms of velocity direction and amplitude. It affects TDE measurements from probes, and is also intrinsically important for beam emission spectroscopy (BES) and gas puff imaging (GPI) measurements. Local eddy shapes estimated from 2D fluctuating field are necessary to gain a more accurate flow estimate from TDE, as illustrated by Langmuir probe array measurements. A least square regression approach is proposed to estimate both flow field and shaping parameters. The technique is applied to a test case built from numerical simulation of interchange fluctuations. The local eddy shape does not only provide corrections for the velocity field, but also quantitative informations about the statistical interaction mechanisms between local eddies and $E \times B$ flow shear. The technique is then tested on gaz puff imaging data collected at the edge of EAST tokamak plasmas. It is shown that poloidal asymmetries of the fluctuation fields - velocity and eddy shape - are consistent at least qualtitatively with a ballooning type of turbulence immersed in a radially sheared equilibrium flow.

a)Electronic mail: nicolas.fedorczak@cea.fr 


\section{INTRODUCTION}

The interplay between large scale flows and local turbulence is an ubiquitous phenomenon of natural and laboratory fluid systems. The organization of zonal flows, known as azimuthally symmetric band-like sheared flows, is a particularly challenging phenomenon for the understanding of dynamo effects, atmospherics jets, or transport regulation in magnetized plasmas ${ }^{1}$. The L$\mathrm{H}$ confinement transition observed at the edge of tokamak plasmas is probably related to the turbulent drive of a sheared zonal flow associated with a strong inward gradient of electric field ${ }^{2-4}$. In addition to the fundamental implications for nonlinearity in fluids, turbulence analysis find direct applications in the optimization of a fusion tokamak reactor performance via the control of turbulent losses mitigated by the plasma rotation. In both cases, the key issue is to then understand how local vortices and eddies created by non-linear instabilities couple to the zonal flows and drive them ${ }^{5}$.

In order to make progress on these physics issues, there are several experimental and theoretical tasks that must be addressed. First, reliable and coherent measurements of flows need to be made using redundant and distinct diagnostics. Second, theory needs to define the relevant fluctuation parameters that should be extracted from data to investigate the interplay between local eddies and large scale flows. Third, techniques to then reliably and robustly extract these quantities from experimental data.

Edge plasmas flows are investigated by a variety of different diagnostics. For instance, reflectometry systems can measure the Doppler phase velocity of fluctuations ${ }^{6}$. Langmuir probes can estimate the drift velocity directly if confidence is accorded to potential measurements ${ }^{7}$. More generally, multidimensional arrays like Beam emission spectroscopy (BES) are used to infer phase velocities by means of cross-correlations ${ }^{8}$. If the array size is large as for fast visible imaging, direct eddy tracking is possible ${ }^{9}$. Otherwise, the methods are limited to time delay estimation (TDE) techniques ${ }^{10,11}$, as for probes ${ }^{12,13}$, beam emission spectroscopy diagnostics ${ }^{8}$ or gas puff imaging (GPI $)^{14}$.

The second point relates to the choice of first principle conservation criterion referred to describe the system. For instance, conservation of momentum links the turbulence and large scale flow via the Reynolds tensor ${ }^{15}$. Measurement of Reynolds stress are possible by Langmuir probes arrays $^{4,16-20}$ and in general data show reasonable coherency with the flow organization. But, little is done to give an interpretation to the existence of this Reynolds stress. Fundamentally, this stress arises from a symmetry breaking in the wave space, which is also to some extents similar to a preferential coupling between fluctuations along two dimensions of space. Said differently, it 
corresponds to a preferential shaping of the vortices ${ }^{5}$. The issue remains to understand the detail of the tilting process. Electrostatic shear could self amplify by favorably tilting the vortices over time ${ }^{21}$, dynamics that should be also pictured on density eddies ${ }^{22}$, and electrostatic particle flux ${ }^{23}$. Besides, magnetic shear could also act on the spacial shape of the eddies ${ }^{14,23}$.

This contribution focuses on statistical methods to interpret time cross-correlations from 2D spatial arrays. These methods are not limited to magnetized plasma experiments and should find also interest in the fluid community. What can be easily and properly extracted from cross correlations on 2D fluctuating fields, regarding velocity fields and eddy shaping informations is the main focus. The first part deals with a proper interpretation of time delays in term of fluctuation velocity field. It is shown that the link between TDE velocity and convective eddy velocity is completely determined by the local statistical shape of the eddies. A straighforward illustration is proposed from data collected by probes arrays in Tore Supra scrape-off layer plasmas ${ }^{24}$. Then, a method is exposed to identify analytic parameters that describe this shape, which are then used to infer the convective eddy velocity from time delays. In a second part, the method is applied to $2 \mathrm{D}$ density fluctuations obtained from simulation, similar to data obtained from fast visible imaging, or probe arrays. It is shown that both poloidal and radial eddy velocity can be reasonably inferred. The statistical orientation of the density eddies is shown to agree with the action of the electrostatic shear. Finally, the technique is applied to gaz puff imaging data collected at the edge of the EAST tokamak $^{25}$ by means of two fast visible camera looking above and below the midplane. Poloidal asymmetries of both eddy shapes and eddy velocity are highlighted by the analysis, which are consistent with a ballooning type of instability.

\section{TIME DELAY ESTIMATION OF EDDY VELOCITY FIELD}

Time delay estimation of velocity is based on the following principle : in the Taylor approximation of locally frozen turbulence pattern advected by a flow, two points aligned along the flow will manifest a time lag $\tau=d / V$ equal to the ratio of the distance $d$ between them to the flow speed $V$. This case is reported on Fig. 1-1. This method is often applied to BES or probes data to infer the zonal flow velocity, although suffering from serious limitations like:

1 the frozen pattern assumption is only a local approximation, since eddies evolve on a time scale known as eddy life time. Thus it is a requirement that the time delay inferred between two points in space be smaller than the eddy life time to write an ubiquitous relationship between time delay and velicity field. 


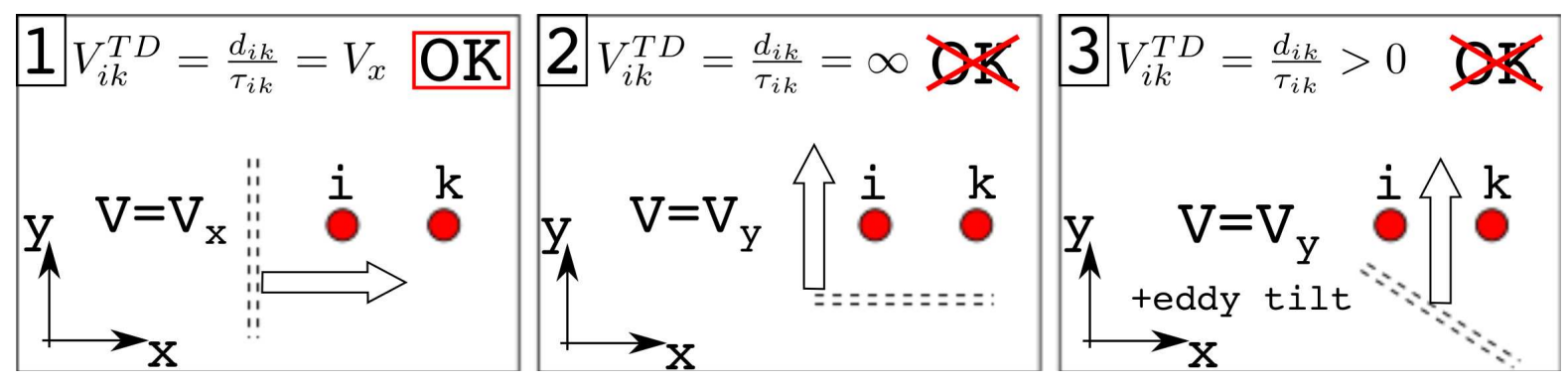

FIG. 1. Illustration of three typical cases encountered in time delay velocity estimation. 1 : the velocity of the fluctuation is aligned with the two points $i$ and $k$, the estimated velocity $V_{i k}$ is right. 2 : the velocity is normal to the points axis, the time delay is zero and the estimated velocity is infinite, which is wrong. 3 : the velocity is normal to the points orientation but the eddies are tilted with respect to their velocity direction, which leads to a finite but artificial velocity along the points axis.

2 the previous relation linking time delay and flow speed is not convincing. Indeed, if the flow is perpendicular to the points alignment as in Fig. 1-2, or if the turbulent eddies present a spacial orientation with respect to the flow as in fig 1-3, which are cases not predictable in advance, the estimated velocity is not equal to the convective eddy velocity

3 the approximation of passive turbulent pattern embedded in a mean flow is not true in magnetized plasma where eddies can present self-generated dynamics created by local polarization effects ( ${ }^{23}$ and references herein). As a thought experiment, let us consider plasma blobs propagating purely radially at the edge of a toroidal plasma device, free of any mean electric field. The radial time delay would correspond to the radial velocity of the eddies, which is not associated to any mean flow. Therefore, any velocity techniques should consider the possible superposition of a mean flow field and an eddy velocity field. This is highlighted in the last part of the paper.

The second point is illustraded by experimental evidences. In Tore Supra scrape-off layer, plasma fluctuations in ohmic discharges have been investigated using two rake probes mounted on toroidally separated reciprocating probe holders ${ }^{23}$. These probes collect fluctuations at the top of scrape-off layer plasmas, by small pins separated by $3 \mathrm{~mm}$. Here time delays are estimated on saturation current fluctuations. The continuous signals collected at a rate of $1 \mathrm{MHz}$ during $200 \mathrm{~ms}$ reciprocations are divided into $4 \mathrm{~ms}$ sub-windows, wich are then cross-correlated between adjacent pins. The maximum of correlation and its respective delay are reported on Fig.2. Over the 6cm of radial profile shown here (the signal drops below the noise level further out), the correlation 

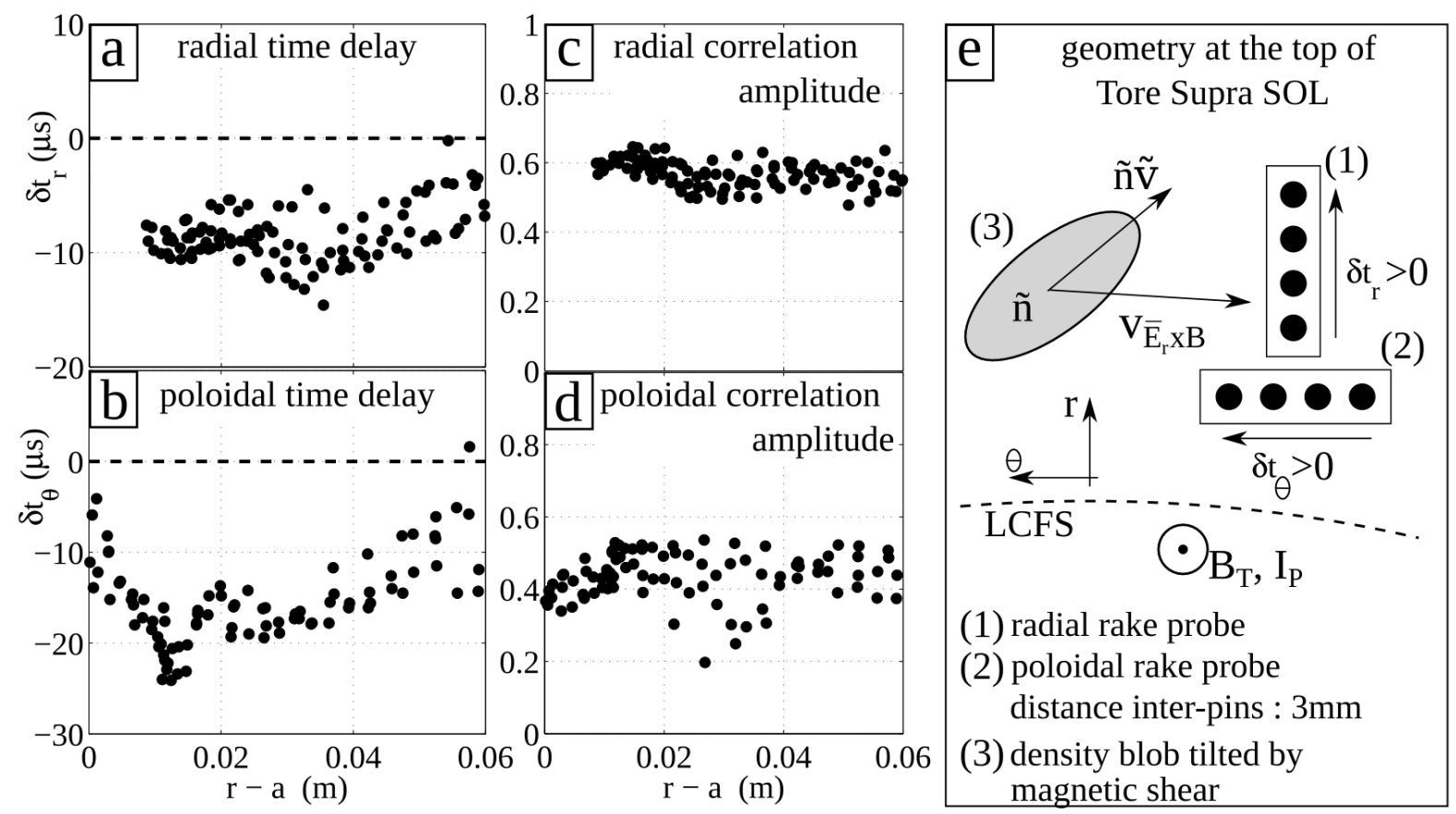

FIG. 2. Experimental data from Tore Supra shot 44635, collected by 2 toroidally separated rake probes mounted on reciprocating holders located at the top of the plasma (e). (a) and (c) are the time delays along the radial and poloidal direction estimated over $4 \mathrm{~ms}$ subwindows by cross-correlation between adjacent pins. (b) and (d) are the respective correlation amplitudes.

amplitude is relatively high (around 0.5), the poloidal time delay is negative (around -10 to -20 $\mu \mathrm{s}$ ) as well as the radial time delay (around $-10 \mu \mathrm{s}$ ). The negative poloidal delay is consistent with a mean scrape-off layer flow directed from the plasma top to the outboard midplane, associated with a mean radial electric field directed outward. On the other hand, the negative radial delay would correspond to density fluctuations propagating inward, although it is well known that the dominant density fluctuations propagate outward in scrape-off layer plasmas ${ }^{23}$. In this later publication, it has been shown that electrostatic fluctuations at the top are consistent with eddies tilted by the magnetic shear, as shown on Fig.2(e). These tilted eddies are embedded in a negative mean poloidal flow, wich amplitude is at least slighly larger than the intrinsic dynamic of the eddies. As a consequence, an eddy crossing the radial rake will do it preferentially with a poloidal dynamic dominated by the mean flow. Given the local magnetic shear tilting, the first pin receiving the perturbation is the radially furthest one, then followed by the radially closest one: the radial time delay is negative. 


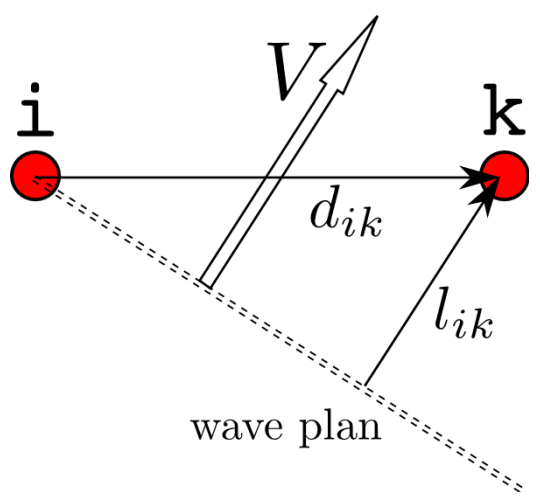

FIG. 3. geometry of two points $(i, k)$ measuring the time delay of a plane wave propagating with the velocity $\mathbf{V}$.

In the following we determine the correct relation between time delays and convective eddy velocity, based on geometrical principles. Effects of eddy orientation on time delays estimation of the velocity field has to be treated in two steps. First, time delays are interpreted in terms of plane wave velocity, and then the relation between plane wave velocity and convective eddy velocity is clarified. Note that in this paper, time delays are estimated using cross-correlations. This implies to use a signal length longer than several turbulent time scales to give a statistical meaning to the correlation. The delay then gives information about the stastistical velocity of the eddyes. It contains the intrinsic eddy dynamics plus the mean flow. Thus if the mean flow is suspected to slowly oscillate in time with a time scale well below the turbulent one, the sub-windows length could be selected to be smaller than the typical variation time scale of the flow but larger than the turbulent time scale in order to resolve the mean flow oscillation. In the following, we assume that both the intrinsic eddy velocity field and mean flow field do not present slow oscillations along time.

\section{A. Velocity of a plane wave}

The most reliable interpretation of time delays in term of velocity field is by considering a plane wave propagating with a velocity normal to its surface (the wave plan). The time delay between two points $(i, k)$ separated by the vector $\mathbf{d}_{i k}$ is equal to the time needed by the wave plan to propagate from $i$ to $k$, therefore estimated along the distance defined by the projection of $\mathbf{d}_{i k}$ along the velocity field :

$$
\tau_{i k}=\frac{\mathbf{d}_{i k} \cdot \mathbf{V}}{V^{2}} \quad \forall(i, k)
$$


This is the correct interpretation of time delay between two points. It effectively explains why the time delay can be zero if the velocity field is normal to the points axis, whereas the velocity is finite. The deconvolution of each of the velocity components therefore requires at least 3 points in space.

In fact once a triplet of collection point $(i, j, k)$ is considered, there is redundancy about delays, since 3 delays can be estimated whereas 2 are enough in principle to extract the velocity field. The more points are considered, the more redundancy increases. The redundancy, in general, goes with slight inconsistencies due to both errors in the technique of estimating the time delays and the approximation of plane wave propagation. These inconsistencies can define a confidence parameter of the velocity measurement. It can be quantified as an output of a least square regression technique applied to the set of time delays, as explained in detail in appendix A.

\section{B. from plane wave to eddy velocity}

The case 3 shown in Fig. 1 considers an eddy as a segment of a wave plan, meaning the eddy has no curved contour. The (convective) eddy velocity is along the $y$ direction, and the eddy is tilted. Two points aligned along the $y$ direction capture a time lag consistent with this velocity, but points aligned along $x$ capture a time lag due to the tilt of the eddy. In fact, the points effectively see a plane wave passing with a velocity normal to its surface, which projection along the eddy velocity direction equals to the convective eddy velocity. 


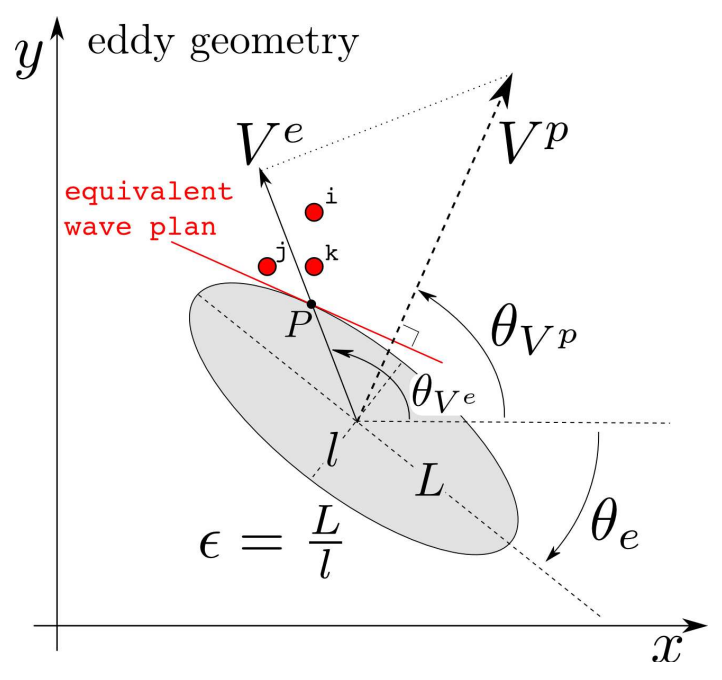

FIG. 4. tilted and shaped eddy propagating with a velocity $V^{e}$. The collection points effectively see an equivalent wave plan propagating with a plane wave velocity $V^{p}$.

Now let us consider a local set of points $(i, j, k, \ldots)$ assumed infinitesimally close to each other. Eddies are considered to behave locally as rigid bodies propagating with constant velocity vectors. Considering the ensemble of eddies passing through the collection points over a statistically relevant time window, we assume that their effects on cross-correlations are equivalent to one effective eddy passing through the collection points. The shape and velocity vector of this eddy are considered to represent the statistical average of these fluctuating fields. In space, the eddy center and the collection points should be aligned on the eddy velocity vector. Then, the infinitesimally closed collection points see only a localised segment of the eddy contour, equivalent to a wave plan. This wave plan is defined geometrically by the tangent surface of the eddy contour at the position where the velocity intercepts the eddy contour, as pictured in Fig.4. In reality, points are not infinitely close, but the effect is statistically identical if we assume that eddies behave with the same dynamics over the local set of collection points.

Once the plane wave velocity $V^{p}$ is estimated from the time delays, and the contour shape of the eddy is known (explained in the following), one can find the angular position $\theta_{V}$ on the eddy contour where the plane wave velocity is normal to the surface, which also defines the orientation of the convective eddy velocity. Its amplitude is then given by the projection of the plane wave velocity on this direction.

This is an important observation for time delay estimation technique in general. Only for turbulence involving isotropic structures is the TDE plane wave velocity equal to the convective eddy velocity. In magnetized plasmas, this assumption is most often not fulfilled since electric shear and 
magnetic shear can significantly participate in tilting and stretching the eddies ${ }^{23,26}$. This result only affects the measurements of the velocity along the direction normal to the eddy velocity. Therefore if the measurement of a zonal flow or other flow within a flux surface is being made, and if this flow dominates the local eddy dynamics, then its measurement is not drastically affected by eddy shaping. On the other hand the radial dynamics of eddies estimated by time delays is completely determined by the shaping effect, as illustrated earlier.

\section{STATISTICAL EDDY SHAPE}

For the study of a given fluctuating field - for instance density- the statistical eddy map refers in the following to the typical amplitude modulation over space of the fluctuations around a reference point. This is calculated by means of cross correlations. We note $f_{c c}\left(x_{1}, x_{2}, \delta t\right)$ the time cross-correlation function between signals collected at $x_{1}$ and $x_{2}$ evaluated at the lag time $\delta t$. Let us consider a $1 \mathrm{D}$ propagation of a cosine wave : $s(x, t)=A_{0} \cos (k(x-v t))$, so that $f_{c c}\left(x_{1}, x_{2}, \delta t\right)=2\left\langle\cos \left(k\left(x_{1}-v(t-\delta t)\right)\right) \cos \left(k\left(x_{2}-v t\right)\right)\right\rangle_{t}=\cos \left(k\left(x_{1}-x_{2}+v \delta t\right)\right)$. Therefore, $f_{c c}\left(x_{0}, x_{0}+\delta x, 0\right)=\cos \left(k \delta_{x}\right)$, which describes the statistical spatial shape of the structure around the position $x_{0}$. In other words, the statistical eddy map $S_{i_{0}}$ defined around the location $i_{0}$ is given by the time cross correlation of the signal taken at this position with the signals taken in its surrounding: $S_{i 0}(x)=f_{c c}\left(x_{i_{0}}, x+x_{i_{0}}, 0\right)$, which can be extended to more than one dimensions in space $^{27,28}$, as illustrated on figure 5 .

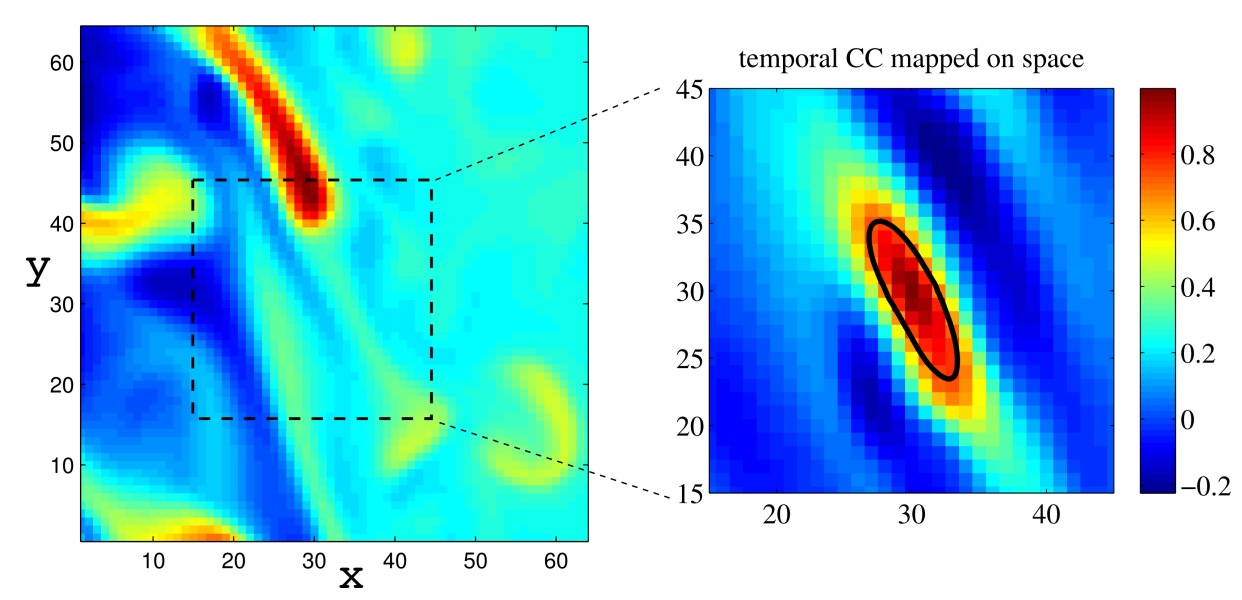

FIG. 5. left : snapshot of a 2D fluctuating density field. right : cross correlation map $S_{i 0}(x, y)$ at a given location $i_{0}$ showing the statistical eddy in color-scale, and estimated contour (black curve) at $S_{i 0}=0.7$.

Once the eddy map is estimated by the cross correlation, it is necessary to define its iso-contours 
to estimate the convective eddy velocity from the TDE plan velocity. This can be difficult if these contours are random. In practice, eddies have often convex structures, and to simplify, presenting an ellipse like contour (see figure 5). As a matter of facts, the approximation is often made that spatial or temporal correlation functions are of Lorenztian or Gaussian type ${ }^{29}$, because experimental evidence exhibits qualitative similarities to these approximations and it is convenient to estimate a statistical width. Considering structure widths different along the horizontal and vertical direction then gives an ellipsoid contour. This elliptical representation gives an analytical link between TDE plane wave and convective eddy velocities. Another physically interesting representation of an eddy shape is by trigonometric functions, for instance $S_{0}(x, y)=\cos (\lambda x) \cos \left(k_{x} x+k_{y} y\right)$, where $k_{y}$ and $k_{x}$ define the orientation of the eddy, and $\lambda$ its horizontal extent. These two representations have an equivalent parabolic expression around the eddy center:

$$
\begin{aligned}
S_{0}(x, y) & =1-\frac{1}{2}\left[\left(\lambda^{2}+k_{x}^{2}\right) x^{2}+k_{y}^{2} y^{2}+2 k_{x} k_{y} x y\right] \\
& =1-\frac{\cos ^{2} \theta_{e}}{\Delta^{2}}\left[\left(1+\epsilon^{2} \tan ^{2} \theta_{e}\right) x^{2}+\left(\epsilon^{2}+\tan ^{2} \theta_{e}\right) y^{2}+2 \tan \theta_{e}\left(1-\epsilon^{2}\right) x y\right]
\end{aligned}
$$

where $\Delta$ is a Gaussian spatial extent of the eddy - along its main axis for the elliptic representation, $\epsilon$ is its aspect ratio and $\theta_{e}$ its orientation (see figure 4). The trigonometric or elliptic parameters of the eddy can be estimated from the statistical eddy map measured by correlations, again using a least square regression method detailed in appendix B.

With the elliptical parameters of the eddy known, the estimation of the convective eddy velocity from the plan wave velocity is relatively easy. In the coordinate system $\left(x^{\prime}, y^{\prime}, \theta^{\prime}\right)$ of the eddy $\left(x^{\prime}\right.$ along the main ellipse axis), the angular position where a vector $u\left(u_{x^{\prime}}, u_{y^{\prime}}\right)$ is normal to the ellipse contour is $\theta_{P_{u}}^{\prime}=\operatorname{atan}\left(\epsilon^{2} u_{x^{\prime}}, u_{y^{\prime}}\right)$, where

$$
\operatorname{atan}(x, y)=\tan ^{-1}\left(\frac{y}{x}\right)+\left\{\begin{array}{lll}
0 & \text { if } & x \geq 0 \\
\pi & \text { if } & x<0
\end{array}\right.
$$

Applying this to the plane wave velocity in the reference coordinate system, the orientation of the eddy velocity is therefore:

$$
\theta_{V^{e}}=\theta_{e}+\operatorname{atan}\left(\epsilon^{2} \cos \left(\theta_{V^{p}}-\theta_{e}\right), \sin \left(\theta_{V^{p}}-\theta_{e}\right)\right)
$$

and its amplitude is :

$$
\left|V^{e}\right|=\cos \left(\theta_{V^{e}}-\theta_{V^{p}}\right)\left|V^{p}\right|
$$


TDE plan wave velocity

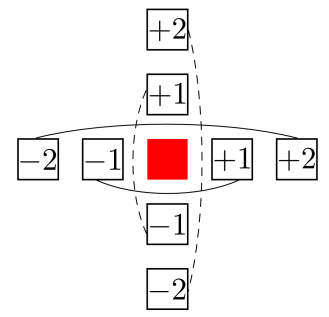

elliptical shape

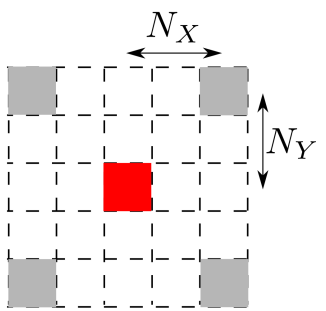

FIG. 6. spatial arrangements of points around a reference for TDE on left and elliptical parameters on right, with $N_{X}=N_{Y}=4$.

\section{ILLUSTRATION ON SIMULATION DATA}

The methods are now tested on a 2D $(x, y)$ density fluctuation field obtained with TOKAM2D, a flux driven scrape-off layer interchange solver for density and potential fields at the outboard midplane $^{30}$. The code has proven to render generic observations of pseudo-ballistic propagation of density blobs, transport attenuation by shear layers, etc. A radial (x) gradient of $E \times B$ poloidal (y) velocity is imposed to the simulation and the zonal potential perturbation is actively forced to this profile. A box of $64 \times 64$ pixels with resolution of 2 hybrid Larmor radius is considered $(\approx 1 \mathrm{~mm})$, and 768 time steps with a resolution of 40 ion larmor periods $(\approx 0.3 \mu \mathrm{s})$. This duration represents about 40 correlation periods of the local turbulence. The regression analysis are performed on set of points as shown in Fig.6, over sub-windows of 256 frames, and output are then averaged over the set of sub-windows. The geometrical schemes shown in Fig.6 are rather simple, indeed in order to provide a minimalist set of correlation information as could be encountered in experimental conditions.

\section{A. Velocity profiles}

Results are shown in Fig.7. The two methods, i.e. plan wave velocity from time delay and convective eddy velocity corrected from the eddy tilt, are compared to the $E \times B$ velocities measured from the plasma potential field. For the radial direction, we considered an effective velocity defined by

$$
v_{x}^{e f f}=\frac{\left\langle\tilde{n} \tilde{v_{x}}\right\rangle_{t}}{\sqrt{\left\langle\tilde{n}^{2}\right\rangle_{t}}}
$$

where $\tilde{n}$ is the density fluctuation (time average subtracted), and $\tilde{v}_{x}=-\partial_{y} \tilde{\Phi}$ where $\Phi$ is the local plasma potential. For the vertical velocity, we consider the time averaged $E_{x} \times B$ potential profile 
imposed in the simulation plus an effective non linear vertical velocity equivalent to the radial one. The definition of $v_{x}^{e f f}$ (or equivalently $v_{y}^{e f f}$ ) is one statistical representation of the $E \times B$ velocity of the density eddies. It is somehow equivalent to the standard deviation of the velocity corrected by the phase shift between density and velocity fluctuations. This definition can not be completely equivalent to the velocity inferred from time delays because delays do not have a simple statistical representation from density and drift velocity fluctuations. But it should be of the same sign and same magnitude.

As shown on Fig.7, the regression analysis gives relatively low values of the least square factors, meaning that the plane wave velocity and elliptical eddy parameters are statistically relevant. The poloidal $E \times B$ velocity profile is relatively well reproduced by the TDE technique. Even the plane wave component is in agreement with the $E \times B$ profile because the velocity field is mostly aligned with the poloidal direction (small radial velocity) lowering the effect of the eddy tilt (as for the case 3 on Fig.1). On the other hand the tilt clearly affects the radial velocity component. Indeed, the radial component of the plane wave velocity reverses sign in the right part of the profile, which is not consistent with the outward $E \times B$ motion of the density blobs. It results from the projection of the poloidal velocity on the radial axis by the eddy tilt. That said, the corrected velocity reproduces the $E \times B$ velocity with a better accuracy. Its sign and amplitude are almost correctly reproduced except for a given radial location where the poloidal $E \times B$ shear is at its maximum. In fact the data show that at that particular position the radial transport efficiency is reduced, only the strongest density events propagate through, which in turn may change the statistical relation of the time delays with density and velocity fluctuations compared to the effective radial velocity. This is an intrinsic limitation of time delay estimation techniques that cannot capture the same events as statistical methods on the $E \times B$ drifts.

\section{B. Eddy shaping}

The eddy regression analysis also provides informations about the tilting of eddies by the flow shear. The elliptic representation is convenient for correcting the velocity field, but effects of $E \times B$ shear on the eddy orientation is given by the trigonometric representation, namely by the horizontal $k_{x}$ and vertical $k_{y}$ wave numbers. The generic idea is that eddies evolving in a background shear flow will be deformed by the flow. Within the trigonometric description, it corresponds to a

continuous evolution of the horizontal wave number $21,23,31: k_{x}(\delta t)=-k_{y} \partial_{x} V_{y} \delta t$ where $\partial_{x} V_{y}$ is the local flow shear and $\delta t$ the local interaction time. Note that other shearing mechanisms can also 

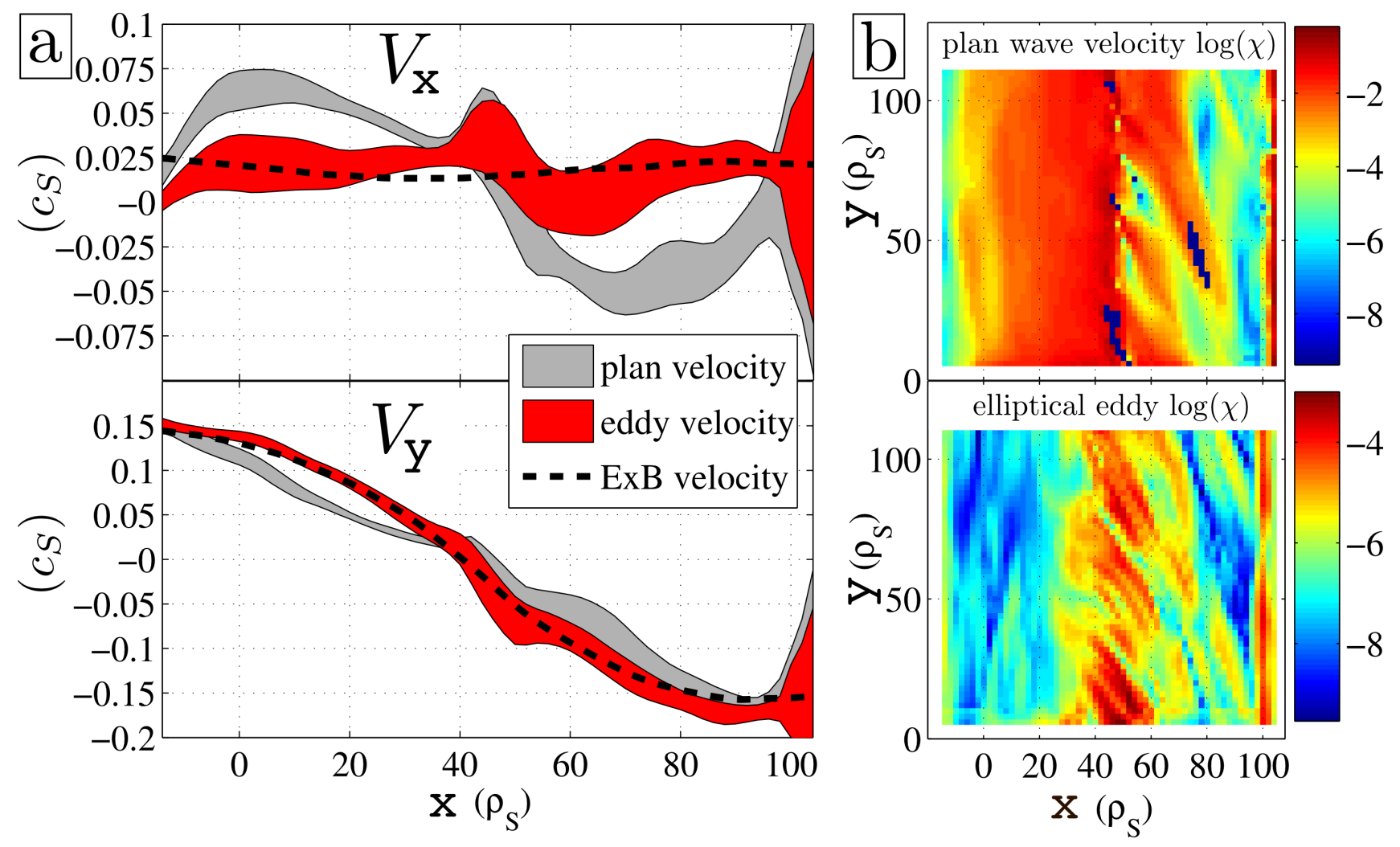

FIG. 7. a: radial and vertical velocity estimated by time delays. The vertical width of the curve represent the standard deviation of the fields along the vertical direction. Grey curves show the plan wave components, and red curves the elliptical correction. The dash curves are the electrostatic drifts components. b: Least square regression factors (log) for the plan wave velocity field and the elliptical representation of the eddies.

influence the relation linking $k_{x}$ to $k_{y}$, as for instance the magnetic shear ${ }^{23}$. Statistically, the previous relation should hold through a statistical average over the interaction time. In Fig. 8 the radial profiles of the zonal shear together with the trigonometric orientation parameter $k_{x} / k_{y}$ are plotted. Clearly, the sign and amplitude of the trigonometric eddy orientation follows the opposite of the shear through the entire profile, as expected from the above considerations. Their ratio gives the local value of the statistical interaction time of the eddies in the shear flow. The critical interest of this approach of interaction time scale is that it is not influenced by the propagation time that normally dominates the auto-correlation time width of fluctuating signal. It rather focus directly on the time scale needed by the shear to tilt the eddies, which is the relevant parameter to characterize the eddy-shear interaction mechanisms. The detailed study of this time scale is beyond the scope of this paper, and will be addressed in dedicated future works. 


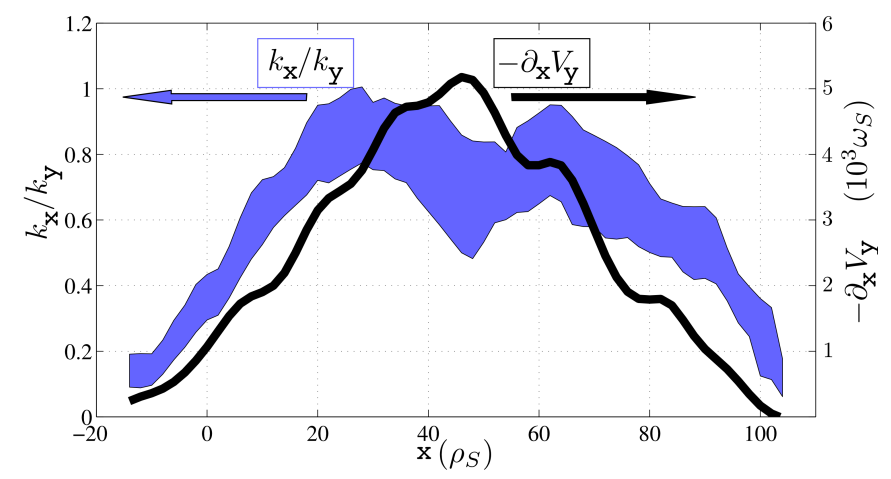

FIG. 8. Profile of the statistical wave number ratio $\left(k_{x} / k_{y}\right)$, with the poloidal deviation shown by the filled area. The black curve is the mean electrostatic shear imposed in the simulation.

\section{APPLICATION TO GAZ PUFF IMAGING DATA}

The technique is now applied to gaz puff imaging data collected at the edge of an EAST tokamak. The GPI system consists of two optical views looking tangentially at the separatrix $\pm 50^{\circ}$ above and below the outboard midplane ${ }^{32}$ (see Fig.9-a). Helium neutrals are injected by gaz nozzles located about $12 \mathrm{~cm}$ from the separatrix. Plasma conditions studied here are : double null L-mode plasma, plasma current $I_{P}=400 \mathrm{kA}$ directed counter-clockwise seen from above, toroidal magnetic field on axis $B_{T}=2.4 \mathrm{~T}$ directed clockwise, low line averaged density $n_{e}=1.510^{19} \mathrm{~m}^{-3}$, weak lower hybrid wave heating $P_{L H}=500 \mathrm{~kW}$. The GPI acquisition is made at a rate of $200 \mathrm{kHz}$, on $64 \times 64$ pixels covering about $12 \mathrm{~cm} \times 12 \mathrm{~cm}$ for each view. A time window of 4096 frames is selected on the stationnary phase of the gaz puff, and fluctuations in the range $10 \mathrm{kHz}<f<100 \mathrm{kHz}$ are considered. Shown in Fig.9-b are the 2D maps of the rms-fluctuation amplitue for each view, in colorscale. The maximum amplitude has been normalized to 1 . Note that in these plasma conditions the visible light emission is rather low, and fluctuations above a noise threshold are spacially limited to a narrow band in the radial direction (of about $3 \mathrm{~cm}$ ), elongated along the flux surface.

The TDE velocity technique is applied on sub-windows of 512 frames. Correlations are performed locally on the same scheme as shown in Fig.6 with $N_{R}=N_{Z}=2$. Then, eddy fields (shape and velocity) are averaged over the set of sub-windows. The velocity field is overplotted on the $2 \mathrm{D}$ maps in Fig.9-b, as well as an exemple of the local eddy shape (black ellipse). The (R,Z) fields are then mapped to the local $(r, \theta)$ coordinates, and averaged along the poloidal direction (the standard deviation of a field along the poloidal direction defines an errorbar in the following). Note that the exact location of the separatrix is not known so that the minor radius coordinates in 
Fig.9-c,d,e,f are only relative positions. This position is adjusted on one view so that both profiles of fluctuation amplitude overlap.
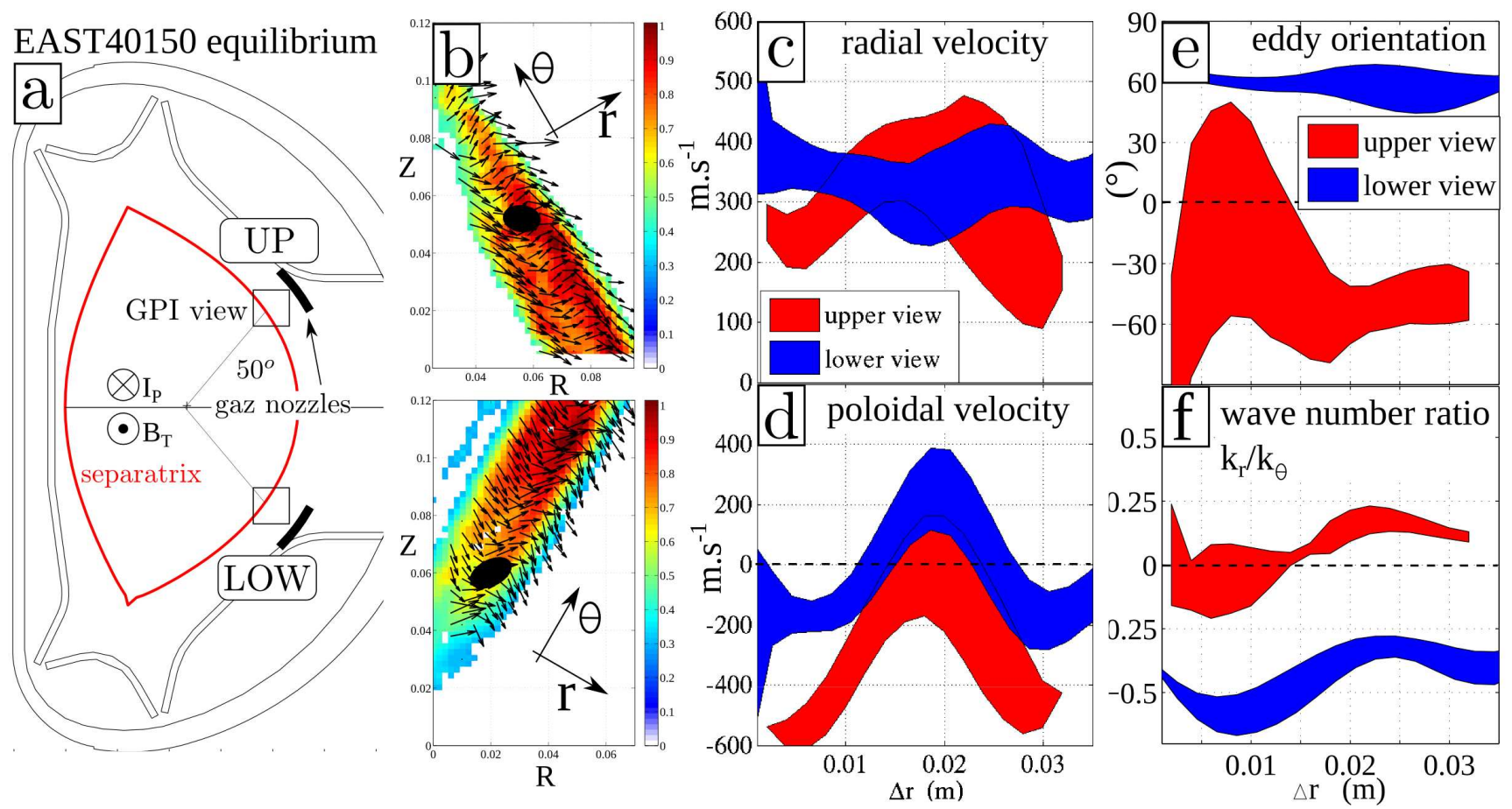

FIG. 9. a) EAST cross section and GPI implementation. b) 2D maps of the fluctuation amplitude collected by GPI, velocity field and eddy shape. c) radial profiles of the poloidally averaged radial velocity of the eddies, in blue for the lower view and red for the upper view. The width of the curve describes the poloidal dispersion of the data. d) poloidal velocity profiles. e) local eddy orientation relative to the normal to the flux surface. f) Eddy orientation paramerized by its wave number ratio.

The radial velocity of the fluctuations is found about 300 to $400 \mathrm{~m} . \mathrm{s}^{-1}$ on both views (Fig.9c), almost overlapping within the errorbar. The poloidal velocities are in the range of -600 to $+400 \mathrm{~m} . \mathrm{s}^{-1}$ (see Fig.9-c), with a velocity measured at the lower view about $300 \mathrm{~m} . \mathrm{s}^{-1}$ higher than the one measured at the upper view. The eddy shape also exhibits up/down differences. For both views, the elliptic aspect ratio of the eddies is about 1.5 and their radius (along the main elliptic axis) about 10mm, but their orientations are reversed (see Fig.9-e). Relative to the normal to the flux surface, eddies at the top are oriented downward $\left(\theta_{e}=-50^{\circ}\right)$, and oriented upward $\left(\theta_{e}=+60^{\circ}\right)$ at the bottom.

These poloidal asymmetries could be an artifact of the TDE technique, althoug always present in results obtained from similar plasmas conditions. In fact, they agree at least qualitatively with a simple model of ballooning turbulence, as proposed in ${ }^{14,23,31}$. Due to the magnetic shear 
first, plasma eddies born from a ballooning instability will exhibit both orientation and poloidal dynamics with an up/down anti-symmetry (on purely symmetric flux surface as for a DN plasma). In addition, the local $E \times B$ shear also participates in the orientation, breaking the pure antisymmerty. The wave vector relation given in section IV B can be exdented including the effect of magnetic shear $\hat{s}^{23,31}$ :

$$
\frac{k_{r}}{k_{\theta}}(\theta)=-\partial_{r} V_{E} \delta t+\hat{s} \theta
$$

where $\theta$ is the poloidal position on the flux surface. As shown in Fig.9-f, the wave number ratio is higher on the upper view with a rather constant offset from the lower view curve. This is in qualitative agreement with the $\theta$ dependance in Eq.4. A quantitative matching requires $\hat{s} \approx 0.34$ which is probably too low for an outboard edge magnetic shear. Nevertheless, the difference in poloidal velocity also agrees qualtitatively with this model. The fluctuation velocity can be written as a superositoin of an equilibrium flow $V_{E}$ and an intrinsic fluctuations velocity due to the projection of the ballooning radial velocity along the poloidal direction (following Eq.4) $v_{\theta}=V_{E}+v_{r}\left(\partial_{r} V_{E} \delta t-\hat{s} \theta\right)$. The velocity offset between the top and the bottom view should be in the order of the radial velocity amplitude.

A quantitative understanding of the results exposed here is uncertain. In fact, the low signal over noise ratio of the data could be the main reason, impacting the cross-correlations and hence the extraction of the eddy shape parameters. Nevertheless, the qualitative agreement with a ballooning type of fluctuations show that the poloidal velocity field dis in fact a superposition of a mean flow field and an eddy velocity field, the later beeing asymmetric poloidally.

\section{CONCLUSION}

Cross correlations are often applied to analysis arrays measurements, like for BES, probes, or fast imaging diagnostics. Interpretation of time delays and spatial contours of correlation amplitudes can bring relevant informations about the local flow speed and the dynamical interaction mechanism between large scale flows and local eddies. A proper link between time delays and statistical eddy velocity is proposed. A least square regression method is put forward to extract from experimental data the eddy velocity field from time delays by considering the effect of statistical eddy shaping on the delays. Not considering the eddy shape in the interpretation of time delays lead to erroneous velocity values, especially in the direction normal to the main flow. This fact is demonstrated on Langmuir probe data collected at the top of Tore Supra SOL plasmas.

The method is tested on 2D density fluctuations from simulations. Density eddies propagate ra- 
dially outward via interchange, through an imposed $E \times B$ shear flow. The shape of the density eddies is observed to evolve in response to the shear flow. As a result, the vertical advection of these tilted eddies by the mean flow creates finite time delays along the radial direction which are not consistent with the actual radial velocity of these eddies. Correcting the time delays velocity technique by including the orientation of the eddies returns a velocity field in closer agreement with the real $E \times B$ velocity field of the fluctuations. Then, the method is tested on GPI data from the EAST tokamak. Both views above and below the midplane are treated simultaneously. It is shown that poloidal dynamics changes from above to below the midplane, as well as the eddy shape. The experimental findings are in qualitative agreement with fluctuations arising from a ballooning instability.

The method can be easily applied to density fluctuations measured by BES, probe arrays and fast imaging, as soon as the number of points in space is not too low. The critical limitation appears for the eddy map, where a minimum of points is required to converge the analytical approximation. Compared to other methods, this one is relatively fast and generic in the application. And besides providing an estimate of the velocity field, it also brings important informations about the dynamic of eddies in a shear flow which should find important application for the experimental investigation of L-H transition using imaging diagnostics.

\section{ACKNOWLEDGEMENT}

This work was supported by the United States D.O.E. grant no. DE-SC0001961. The authors are grateful to the participants of Festival de théorie 2011, organized by the C.E.A of France, for many valuable discussions.

\section{Appendix A: Least square regression of the plan wave velocity}

In the following a pair of points is indexed by $i$, and we consider an ensemble $E$ of pairs $(j k)_{i}, i \in E$, with at least two pairs in the ensemble. For a pair $i$, the vector linking the two points is noted $d_{i}$, the time delay $\tau_{i}$ and the maximum correlation amplitude $A_{i}$. Here we assume that the time delay is estimated by cross-correlation methods, defined as the time lag for which the cross correlation reaches its maximum. The least square factor $\chi$ is defined as the ensemble 
average of the distances between each delay and its relation to the velocity field:

$$
\chi^{V}=\frac{1}{\sum_{i} A_{i} \tau_{i}^{2}} \sum_{i} A_{i}\left[\frac{d_{i} \cdot V}{V^{2}}-\tau_{i}\right]^{2}
$$

This parameter is always positive and cancels if all time delays are perfectly consistent with the velocity orientation (from the relation Eq.1). Note that informations are weighted by the degree of correlation between two points, which could be modified by replacing $A_{i}$ by $A_{i}^{\alpha}$ with $\alpha>0$. The velocity field is then defined from the time delays by minimizing $\chi^{V}$. In the following we consider two directions normal in space, $x$ and $y$, and the velocity is replaced by the vector $u=V /|V|^{2}$. The vector components $\left(u_{x}, u_{y}\right)$ are solutions of the variational expression :

$$
\frac{\partial \chi^{V}}{\partial u_{x}}=0, \quad \frac{\partial \chi^{V}}{\partial u_{y}}=0
$$

, which reads:

$$
\left(\begin{array}{c}
u_{x} \\
u_{y}
\end{array}\right)=\left[\begin{array}{cc}
\sum_{i} A_{i} d_{i, x}^{2} & \sum_{i} A_{i} d_{i, x} d_{i, y} \\
\sum_{i} A_{i} d_{i, x} d_{i, y} & \sum_{i} A_{i} d_{i, y}^{2}
\end{array}\right]^{-1}\left(\begin{array}{c}
\sum_{i} A_{i} d_{i, x} \tau_{i} \\
\sum_{i} A_{i} d_{i, y} \tau_{i}
\end{array}\right)
$$

The main interest of the method stands in the fact that their is no need of special locations of the points as soon as they form a 2D surface. Secondly, by re-inserting the value of $u$ estimated by Eq. A2 in the Eq. A1, we have an estimate of the least-square regression factor of the velocity estimation.

\section{Appendix B: Least square regression of the eddy shape}

In the following we consider $S(x, y)$ the correlation map of the fluctuating field around a reference position $x=0, y=0$, and $f(x, y)$ the parabolic representation of the map :

$$
\begin{aligned}
f(x, y) & =1-\frac{1}{2}\left[\left(\lambda^{2}+k_{x}^{2}\right) x^{2}+k_{y}^{2} y^{2}+2 k_{x} k_{y} x y\right] \\
& =1-\frac{\cos ^{2} \theta_{e}}{\Delta^{2}}\left[\left(1+\epsilon^{2} \tan ^{2} \theta_{e}\right) x^{2}+\left(\epsilon^{2}+\tan ^{2} \theta_{e}\right) y^{2}+2 \tan \theta_{e}\left(1-\epsilon^{2}\right) x y\right]
\end{aligned}
$$

The square parameter to minimize is defined as:

$$
\chi^{e}=\frac{1}{N} \sum_{i}\left[S\left(x_{i}, y_{i}\right)-f\left(x_{i}, y_{i}\right)\right]^{2}
$$

Note that the set of points $(x, y)$ to be considered here should be concentrated around the reference since the parabolic approximation is only local, and should contain at least 4 points reference 
included. The minimization is done on the parabolic parameters rewritten as : $a_{x}=\lambda^{2}+k_{x}^{2}=$ $2 \frac{\cos ^{2} \theta_{e}}{\Delta^{2}}\left(1+\epsilon^{2} \tan ^{2} \theta_{e}\right), a_{y}=k_{y}^{2}=2 \frac{\cos ^{2} \theta_{e}}{\Delta^{2}}\left(\epsilon^{2}+\tan ^{2} \theta_{e}\right)$ and $a_{x y}=k_{x} k_{y}=2 \frac{\cos ^{2} \theta_{e}}{\Delta^{2}} \tan \theta_{e}\left(1-\epsilon^{2}\right)$. The variational method is :

$$
\frac{\partial \chi^{e}}{\partial a_{x}}=0, \quad \frac{\partial \chi^{e}}{\partial a_{y}}=0, \quad \frac{\partial \chi^{e}}{\partial a_{x y}}=0
$$

, which gives the system:

$$
\left(\begin{array}{l}
a_{x} \\
a_{y} \\
a_{x y}
\end{array}\right)=\left[\begin{array}{ccc}
\sum_{i} x_{i}^{4} & \sum_{i} x_{i}^{2} y_{i}^{2} & 2 \sum_{i} x_{i}^{3} i_{k} \\
\sum_{i} x_{i}^{2} y_{i}^{2} & \sum_{i} y_{i}^{4} & 2 \sum_{i} x_{i} y_{i}^{3} \\
\sum_{i} x_{i}^{3} y_{i} & \sum_{i} x_{i} y_{i}^{3} & 2 \sum_{i} x_{i}^{2} y_{i}^{2}
\end{array}\right]^{-1}\left(\begin{array}{c}
2 \sum_{i} x_{i}^{2}\left(1-S_{i}\right) \\
2 \sum_{i} y_{i}^{2}\left(1-S_{i}\right) \\
2 \sum_{i} x_{i} y_{i}\left(1-S_{i}\right)
\end{array}\right)
$$

The parameters $\left(\lambda, k_{x}, k_{y}\right)$ are easily expressed from $a_{x}, a_{y}, a_{x y}$, and the elliptic ones need the solve of an other system. We define $a=\frac{a_{x}}{a_{y}}$ and $b=\frac{a_{x y}}{a_{y}}$, so that the system on $\epsilon$ and $\theta_{e}$ becomes :

$$
\begin{aligned}
& \epsilon^{2}=\frac{1-a \tan ^{2} \theta_{e}}{a-\tan ^{2} \theta_{e}} \\
& b \tan ^{4} \theta_{e}+(a-1) \tan ^{3} \theta_{e}+(a-1) \tan \theta_{e}-b=0
\end{aligned}
$$

The tangent of the tilt angle is solution of a forth degree polynomial, and only one solution is real and satisfies $\epsilon \geq 1$. Note that $\theta_{e}$ is defined in $[-\pi / 2, \pi / 2]$.

\section{REFERENCES}

${ }^{1}$ P H Diamond, S-I Itoh, K Itoh, and T S Hahm. Plasma Physics and Controlled Fusion, 47(5):R35, 2005.

${ }^{2}$ M. Xu, G. R. Tynan, P. H. Diamond, P. Manz, C. Holland, N. Fedorczak, S. Chakraborty Thakur, J. H. Yu, K. J. Zhao, J. Q. Dong, J. Cheng, W. Y. Hong, L. W. Yan, Q. W. Yang, X. M. Song, Y. Huang, L. Z. Cai, W. L. Zhong, Z. B. Shi, X. T. Ding, X. R. Duan, and Y. Liu. Phys. Rev. Lett., 108:245001, 2012.

${ }^{3}$ L. Schmitz, L. Zeng, T. L. Rhodes, J. C. Hillesheim, E. J. Doyle, R. J. Groebner, W. A. Peebles, K. H. Burrell, and G. Wang. Phys. Rev. Lett., 108:155002, 2012.

${ }^{4}$ P. Manz, G. S. Xu, B. N. Wan, H. Q. Wang, H. Y. Guo, I. Cziegler, N. Fedorczak, C. Holland, S. H. Müller, S. C. Thakur, M. Xu, K. Miki, P. H. Diamond, and G. R. Tynan. Physics of Plasmas, 19(7):072311, 2012.

${ }^{5}$ P. Manz, M. Ramisch, and U. Stroth. Phys. Rev. Lett., 103:165004, 2009.

${ }^{6}$ P. Hennequin, C. Honor, A. Truc, A. Qumneur, C. Fenzi-Bonizec, C. Bourdelle, X. Garbet, G.T. Hoang, and the Tore Supra team. Nuclear Fusion, 46(9):S771, 2006. 
${ }^{7}$ B. Nold, T. T. Ribeiro, M. Ramisch, Z. Huang, H. W. Mller, B. D. Scott, U. Stroth, and ASDEX Upgrade Team. New journal of Physics, 2011.

${ }^{8}$ G. R. McKee, R. J. Fonck, D. K. Gupta, D. J. Schlossberg, M. W. Shafer, C. Holland, and G. Tynan. Review of Scientific Instruments, 75(10):3490-3492, 2004.

${ }^{9}$ T. Munsat and S. J. Zweben. Review of Scientific Instruments, 77(10):103501, 2006.

${ }^{10}$ C. Holland, G. R. Tynan, G. R. McKee, and R. J. Fonck. Review of Scientific Instruments, 75(10):4278-4280, 2004.

${ }^{11}$ Balazs Tal, Attila Bencze, Sándor Zoletnik, Gabor Veres, and Gabor Por. Physics of Plasmas, 18(12):122304, 2011.

${ }^{12}$ S.C Thakur, D. McCarren, T. Lee, N. Fedorczak, P. Manz, E.E. Scime, G.R. Tynan, and M. Xu. Physics of Plasmas, 19(7), 2012.

${ }^{13}$ S.C. Thakur, D. McCarren, T. Lee, N. Fedorczak, P. Manz, E. E. Scime, G. R. Tynan, M. Xu, and J. Yu. Review of Scientific Instruments, 83(10):10D708, 2012.

${ }^{14}$ J.L. Terry, S.J. Zweben, O. Grulke, M.J. Greenwald, and B. LaBombard. Journal of Nuclear Materials, 3337339:322 - 326, 2005.

${ }^{15}$ P. H. Diamond and Y.-B. Kim. Physics of Fluids B: Plasma Physics, 3(7):1626-1633, 1991.

${ }^{16}$ C. Hidalgo, C. Silva, M. A. Pedrosa, E. Sánchez, H. Fernandes, and C. A. F. Varandas. Phys. Rev. Lett., 83:2203-2205, 1999.

${ }^{17}$ N. Vianello, E. Spada, V. Antoni, M. Spolaore, G. Serianni, G. Regnoli, R. Cavazzana, H. Bergsåker, and J. R. Drake. Phys. Rev. Lett., 94:135001, 2005.

${ }^{18}$ C. Holland, J. H. Yu, A. James, D. Nishijima, M. Shimada, N. Taheri, and G. R. Tynan. Phys. Rev. Lett., 96:195002, 2006.

${ }^{19}$ Z. Yan, J. H. Yu, C. Holland, M. Xu, S. H. Müller, and G. R. Tynan. Physics of Plasmas, 15(9):092309, 2008.

${ }^{20}$ P. Manz, M. Xu, N. Fedorczak, S. C. Thakur, and G. R. Tynan. Physics of Plasmas, 19(1):012309, 2012 .

${ }^{21}$ V S Mikhailenko, V V Mikhailenko, and K N Stepanov. Plasma Physics and Controlled Fusion, 52(5):055007, 2010.

${ }^{22} \mathrm{Ph}$. Ghendrih, G. Ciraolo, Y. Larmande, Y. Sarazin, P. Tamain, P. Beyer, G. Chiavassa, G. Darmet, X. Garbet, and V. Grandgirard. Journal of Nuclear Materials, 390391(0):425 427, 2009 . 
${ }^{23}$ N. Fedorczak, J. P. Gunn, J.-Y. Pascal, Ph. Ghendrih, G. van Oost, P. Monier-Garbet, and G. R. Tynan. Physics of Plasmas, 19(7):072314, 2012.

${ }^{24}$ G. Giruzzi and Tore Supra team. Nuclear Fusion, 49(10):104010, 2009.

${ }^{25}$ Baonian Wan and International Collaborators. Nuclear Fusion, 49(10):104011, 2009.

${ }^{26}$ J.L. Terry, S.J. Zweben, M.V. Umansky, I. Cziegler, O. Grulke, B. LaBombard, and D.P. Stotler. Journal of Nuclear Materials, 390391(0):339 - 342, 2009.

${ }^{27}$ M Ramisch, F Greiner, N Mahdizadeh, K Rahbarnia, and U Stroth. Plasma Physics and Controlled Fusion, 49(6):777, 2007.

${ }^{28}$ M. Ramisch, N. Mahdizadeh, U. Stroth, F. Greiner, C. Lechte, and K. Rahbarnia. Physics of Plasmas, 12(3):032504, 2005.

${ }^{29}$ J. E. Maggs and G. J. Morales. Phys. Rev. Lett., 107:185003, 2011.

${ }^{30}$ Y. Sarazin and Ph. Ghendrih. Physics of Plasmas, 5(12):4214-4228, 1998.

${ }^{31}$ N. Fedorczak, P.H. Diamond, G. Tynan, and P. Manz. Nuclear Fusion, 52(10):103013, 2012.

${ }^{32}$ S.C. Liu. New dual gas puff imaging system with up-down symmetry on east tokamak. Rev. Sci. Instrum., submitted, 2012. 